\title{
磷酸银纳米结构的调控及其光催化性能研究
}

\author{
蔡维维, 李蛟, 何静, 王卫伟 \\ (山东理工大学 材料科学与工程学院, 淄博 255049)
}

摘 要: 基于十二烷基硫酸钠(SDS)、环己烷、异戊醇和水溶液构建的反相微乳液体系, 以硝酸银、磷酸二氢钾为原 料, 通过改变水与表面活性剂物质的量比值 $\left(W\right.$ ) 调控制备出棒状、纺锤状及四面体状磷酸银 $\left(\mathrm{Ag}_{3} \mathrm{PO}_{4}\right)$ 纳米材料。利 用 XRD、SEM、TEM 和 UV-Vis-DRS 光谱对样品结构、形貌以及可见光响应特性进行了表征，探讨了不同形貌 $\mathrm{Ag}_{3} \mathrm{PO}_{4}$ 的形成机理，并以次甲基蓝(MB)降解效果评价了样品光催化性能。实验结果表明: 制备的系列纳米 $\mathrm{Ag}_{3} \mathrm{PO}_{4}$ 均为体心立方结构晶体, 其形貌受 $W$ 值影响明显; $\mathrm{Ag}_{3} \mathrm{PO}_{4}$ 形貌的不同主要由于 $\mathrm{SDS}$ 量的变化改变了含有产物水核 的直径与界面膜强度。此外, 制备的样品均表现出好的可见光催化降解 MB 性能, 其中, 具有完整四面体形貌的 $\mathrm{Ag}_{3} \mathrm{PO}_{4}$ 光催化性能最佳, 这与其能够裸露出多的 $\{111\}$ 晶面有关。

关 键 词: 反相微乳液法; 磷酸银; 形貌; 可见光响应; 光催化性能

中图分类号: O64 文献标识码: A

\section{Controlled Synthesis and Photocatalytic Activity Evaluation of Nanostructured $\mathrm{Ag}_{3} \mathrm{PO}_{4}$}

\author{
CAI Wei-Wei, LI Jiao, HE Jing, WANG Wei-Wei
}

(School of Materials Science and Engineering, Shandong University of Technology, Zibo 255049, China)

\begin{abstract}
Silver phosphate $\left(\mathrm{Ag}_{3} \mathrm{PO}_{4}\right)$ materials with different nanostructures including rod, cambiform and tetrahedron with round edges and corners were synthesized by adjusting the molar ratio $(W)$ of water to surfactant in a reverse microemulsion, which consists of sodium dodecyl sulfate (SDS), isopentyl alcohol, cyclohexane, and aqueous solution, using silver nitrate and potassium dihydrogen phosphate as starting materials. The structure, morphology and visible-light-response of the obtained samples were characterized by XRD, SEM, HRTEM, and UV-Vis-DRS spectra. The formation mechanisms of nanostructured $\mathrm{Ag}_{3} \mathrm{PO}_{4}$ were discussed. Moreover, the photocatalytic activity was also evaluated by means of degradation of organic dye methylene blue (MB) in water. The results show that all the samples have body-centred cubic crystal structure, and the $\mathrm{W}$ value has an obvious effect on morphologly of nanostructured $\mathrm{Ag}_{3} \mathrm{PO}_{4}$. The varied morphologies can be attributed to the change of diameter and interfacial film strength of water nuclear influenced by SDS content. In addition, all the obtained samples exhibit excellent efficient photocatalytic activity for the photo-degradation of methylene blue (MB) under visible light irradiation, and the tetrahedral $\mathrm{Ag}_{3} \mathrm{PO}_{4}$ with exposed many $\{111\}$ facets exhibits the best photocatalytic activity.
\end{abstract}

Key words: reverse phase microemulsion; silver phosphate; nanostructure; visible light response; photocatalytic activity

收稿日期: 2016-05-11; 收到修改稿日期：2016-07-15

基金项目：山东省高等学校科技发展计划项目(J15LA08); 山东理工大学青年教师发展支持计划项目(4072-114019) College Technology Development Project of Shandong Province, China (J15LA08); Support Development Project for Young Teachers of Shandong University of Technology (4072-114019)

作者简介: 蔡维维(1992-), 女, 硕士研究生. E-mail: 767789055@qq.com

通讯作者: 李 蛟, 副教授. E-mail: haiyan9943@163.com 
设计合成具有可见光响应特性的半导体光催化 材料, 提高对太阳光利用率是未来光催化技术的发 展方向。磷酸银(Silver phosphate, $\left.\mathrm{Ag}_{3} \mathrm{PO}_{4}\right)$ 间接禁带宽 度约为 $2.36 \mathrm{eV}$, 在波长小于 $530 \mathrm{~nm}$ 光源照射下, 表 现出强的光氧化能力, 可以有效降解有机污染物, 量子效率超过 $90 \%{ }^{[1]}$, 是一种具有高催化活性的可 见光响应催化材料, 近年来备受研究人员关注。

半导体材料光催化性能与其自身尺寸和形貌结 构密切相关。 $\mathrm{Bi}$ 等 ${ }^{[2]}$ 利用离子交换法控制合成出了 十二面体与立方体形貌的 $\mathrm{Ag}_{3} \mathrm{PO}_{4}$ 粉体, 光催化活 性实验结果显示前者光催化降解甲基橙和罗丹明 $\mathrm{B}$ 的效果明显强于后者。此外, 通过改变离子交换过 程中反应物浓度与种类、反应液 $\mathrm{pH}$ 或添加成核剂 等方式, 也可获得不同光催化活性且形貌各异的 $\mathrm{Ag}_{3} \mathrm{PO}_{4}$ 产物, 如球状、花状、四面体、多面体、短 足状及树枝状等 ${ }^{[3-5]}$ 。尽管离子交换法在制备不同形 貌 $\mathrm{Ag}_{3} \mathrm{PO}_{4}$ 光催化材料方面显示出简单易行的特点, 但制备的纳米粒子存在易团聚、颗粒大和形貌不均 匀等缺陷, 使人们难以准确深入研究 $\mathrm{Ag}_{3} \mathrm{PO}_{4}$ 形貌 结构与光催化性能之间的关系。

微乳液法是一种适用于制备纳米材料的微型软 模板控制技术，具有反应条件温和、实验装置简单 易操作等特点。该法可以通过改变微乳体系中表面 活性剂含量而灵活调节乳液滴直径, 从而精确调控 样品的尺寸和形貌。因此, 在制备尺寸均一、形貌 可控的纳米材料方面显示出传统制备方法无法比拟 的优越性 ${ }^{[6]}$ 。目前, 利用微乳液法已成功制备了 $\mathrm{ZnO}^{[7]} 、 \mathrm{TiO}_{2}{ }^{[8]} 、 \mathrm{WO}_{3}{ }^{[9]}$ 等多种具有光催化活性且形 貌独特的纳米半导体光催化材料, 但基于微乳液法 制备纳米 $\mathrm{Ag}_{3} \mathrm{PO}_{4}$ 的研究工作却鲜有报道, 与之相 关的形貌形成机理研究更未有触及。

本工作拟以十二烷基硫酸钠(SDS)、环己烷、异 戊醇和水溶液构建反相微乳液体系, 以硝酸银、磷 酸二氢钾为原料, 通过改变水与表面活性剂物质的 量比值 $(W)$, 对 $\mathrm{Ag}_{3} \mathrm{PO}_{4}$ 纳米结构实现准确调控。研 究探讨了不同形貌纳米 $\mathrm{Ag}_{3} \mathrm{PO}_{4}$ 的形成机理, 并以 次甲基蓝(MB)降解效果评价样品的光催化性能。

\section{1 实验方法}

\section{1 主要试剂与仪器}

硝酸银 $\left(\mathrm{AgNO}_{3}\right)$, 上海银典化工有限公司; 磷 酸二氢钾 $\left(\mathrm{KH}_{2} \mathrm{PO}_{4}\right)$, 天津市巴斯夫化工有限公司; 十二烷基硫酸钠(Sodium Dodecyl Sulfate, SDS), 次 甲基蓝(Methylene Blue, MB), 天津市福晨化学试剂 厂; 环已烷, 异戊醇均为分析纯, 天津市大茂化学
试剂厂。实验所用蒸馏水采用 EPED-E2-10TJ 型超 纯水器制备。

WH220 型加热磁力搅拌器, 北京桑翌技术公司; HC-2518 型高速离心机, 安徽中科中佳科学仪器有 限公司; HZC-50 型超声波清洗器, 福州华志科学仪 器公司; DZF 型真空干燥箱, 上海科恒实业发展有 限公司; Sirion 200 型场发射扫描电子显微镜(SEM), 荷兰 FEI 公司; D8 Advance 型多晶 X 射线衍射仪 (XRD), 德国 Bruker AXS 公司; FEI-TECNAI G2 型 透射电镜(TEM), 荷兰 FEI 公司; Shimadzu 型 UV-2550 型紫外-可见光谱仪(UV-Vis)，日本岛津公 司; 755PC 型分光光度计, 上海光谱仪器公司; CHF-XM500 型太阳能模拟光源系统，北京畅拓科 技公司; ASAP2460 型全自动比表面积及孔径分析 仪(BET)，美国麦克公司。

\section{2 微乳液法制备纳米磷酸银}

将 $1 \mathrm{~g} \mathrm{SDS}$ 加入 $17.5 \mathrm{~mL}$ 环己烷和 $5 \mathrm{~mL}$ 异戊醇 混合溶液中, 磁力搅拌 $1 \mathrm{~h}$ 后平均分为二份: 一份加 入适量体积一定浓度的 $\mathrm{AgNO}_{3}$ 溶液, 标记微乳液 $\mathrm{A}$; 另一份加入等量相同浓度的 $\mathrm{KH}_{2} \mathrm{PO}_{4}$ 溶液, 标记 微乳液 $\mathrm{B}$ 。将微乳液 $\mathrm{B}$ 逐滴加入微乳液 $\mathrm{A}$ 中, 快速 搅拌 $2 \mathrm{~h}$ 后室温陈化 $24 \mathrm{~h}$; 破乳, 离心, 清洗后, $60^{\circ} \mathrm{C}$ 下干燥 $10 \mathrm{~h}$, 得黄绿色固体产物。为了对产物 形貌进行调控研究, 本实验分别选择 $W$ 为 $10 、 15$ 、 20、25 的微乳体系制备 $\mathrm{Ag}_{3} \mathrm{PO}_{4}$ 。

\section{3 催化剂的表征}

利用 XRD 鉴定样品的物相结构, $\mathrm{Cu}$ 靶, $\mathrm{K} \alpha$ 为辐 射源, $\lambda=0.15418 \mathrm{~nm}$, 管电压 $40 \mathrm{kV}$; 管电流 $50 \mathrm{~mA}$, 扫描速度 $1 \% \mathrm{~min}$ 。采用 SEM 与 TEM 对样品微观形 貌结构进行分析。采用 UV-Vis 光谱仪测定样品 UV-Vis 吸收光谱, 扫描范围 200 800 nm, 标准 $\mathrm{BaSO}_{4}$ 参比。采用 BET 测量样品的比表面积 $\left(S_{\mathrm{BET}}\right)$ 。

\section{4 光催化活性测定}

将一定量 $\mathrm{Ag}_{3} \mathrm{PO}_{4}$ 加入 $300 \mathrm{~mL} 10 \mathrm{mg} / \mathrm{L}$ 次 $\mathrm{MB}$ 溶液中, 避光条件下吸附一脱附平衡后 $(10 \mathrm{~min})$ 进行 模拟太阳光源照射，每隔一定时间取样，高速离心 分离后取上层清液用分光光度计在 $666 \mathrm{~nm}$ 波长下 检测 $\mathrm{MB}$ 吸光度, 结合 $\mathrm{MB}$ 浓度-吸光度工作曲线计 算出降解后 $\mathrm{MB}$ 浓度 $C_{x}$, 计算 $\mathrm{MB}$ 残留率 $C_{x} / C_{0}$ 以 评价产物的光催化活性, $C_{0}$ 为 $\mathrm{MB}$ 初始浓度。

\section{2 结果与讨论}

\section{1 物相分析}

不同 $W$ 值条件下制备的 $\mathrm{Ag}_{3} \mathrm{PO}_{4}$ 产物 XRD 图谱 如图 1 所示, 对比分析可以看出，实验制备的系列 
样品都具有体心立方结构 $\mathrm{Ag}_{3} \mathrm{PO}_{4}(\mathrm{PDF}$ 06-0505) (110)、(200)、(210)、(211)、(220)、(310)、(222)、 (320)、(321)和(400)晶面的特征衍射峰, 因此可以确 定制备样品均为体心立方结构 $\mathrm{Ag}_{3} \mathrm{PO}_{4}$ 晶体。从图 1 还可以看出, 样品的衍射峰均较为尖锐, 表明制备 的样品均有较好的结晶度。随着 $W$ 值的增大, 样品 衍射峰强度明显增强，表明表面活性剂的减少有利 于样品晶化度的提高。基于谢乐公式计算得到的样 品晶粒尺寸分别为 $41.02 、 37.81 、 39.63 、 38.52 \mathrm{~nm}$, 说 明不同样品晶粒尺寸变化不大。从图 1 还可以看出, 系列样品在 $2 \theta=31.4^{\circ} 、 45.1^{\circ}$ 处均出现强度较弱的衍 射峰, 说明产物中含有少量杂质, 这可能与微乳液 滴内反应物反应不完全有关 ${ }^{[10]}$ 。

\section{2 形貌与结构特征分析}

图 2 为 $\mathrm{Ag}_{3} \mathrm{PO}_{4}$ 产物的 SEM 照片, 由图可见, 随 着 $W$ 值的增大, 样品形貌变化明显。其中, $W=10$ 制 备的样品为长约 $1 \mu \mathrm{m}$, 直径约 $150 \mathrm{~nm}$ 的棒状结构

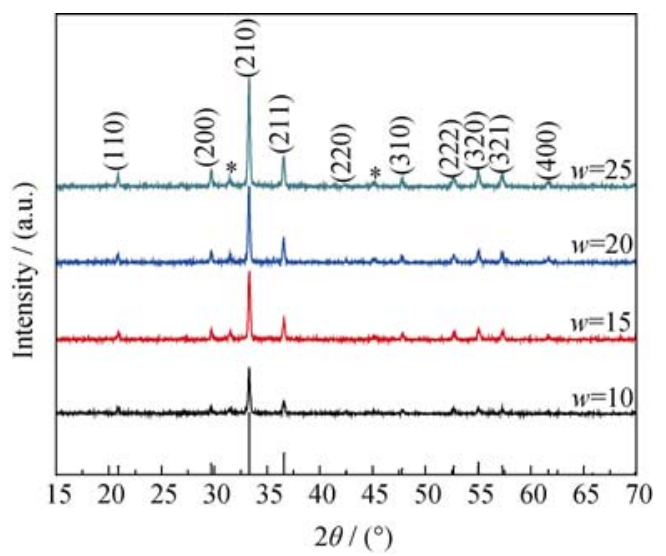

图 1 不同 $W$ (水与表面活性剂的比值)值下制备样品 XRD 图谱 Fig. 1 XRD patterns of $\mathrm{Ag}_{3} \mathrm{PO}_{4}$ nanomaterials prepared with different $W$ values
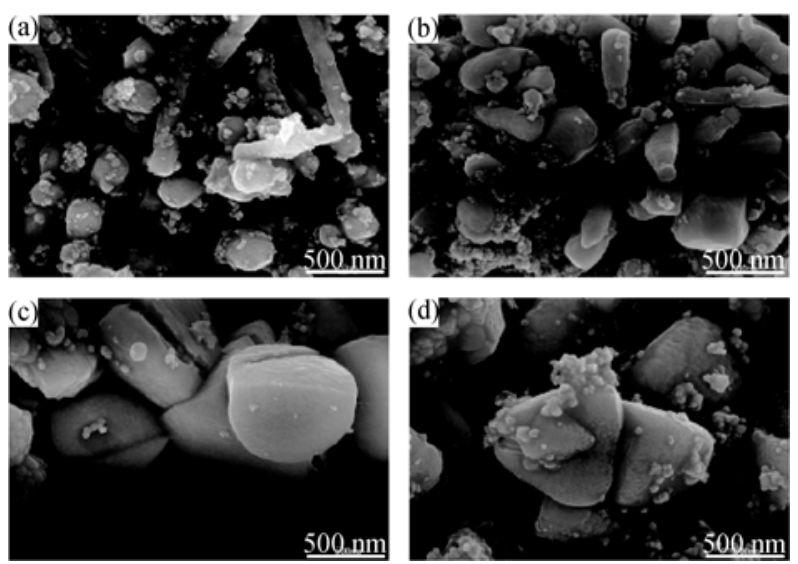

图 2 不同 $W$ (水与表面活性剂的比值)值下制备样品的 SEM 照片 Fig. 2 SEM images of $\mathrm{Ag}_{3} \mathrm{PO}_{4}$ nanomaterials prepared with different $W$ values

(a) $W=10$; (b) $W=15$; (c) $W=20$; (d) $W=25$
(图 2(a)); $W=15$ 制备的样品则表现为长约 $400 \mathrm{~nm}$, 直径约 $190 \mathrm{~nm}$ 的纺锤状结构(图 2(b)); 而 $W=20$ 与 25 制备的样品均具有四面体形貌特征，前者未完全 形成完整的四面体形貌，表面存在明显的缝隙(图 2(c)), 而后者已形成了边缘平滑、四角圆润的正四 面体形貌(图 2(d))。

图3(a) 是具有完整四面体形貌样品的 TEM 照片, 插图为局部区域高分辨照片, 由图可见，该样品由 一定数量球状纳米颗粒相互吸附聚集而成, 图中黑 白区域分布不均匀说明 $\mathrm{Ag}_{3} \mathrm{PO}_{4}$ 纳米颗粒聚集具有 一定随机性, 样品内部呈现出疏松性结构特点。图 3(b) 是粒径约为 $25 \mathrm{~nm} \mathrm{Ag}_{3} \mathrm{PO}_{4}$ 纳米颗粒高分辨 TEM (HRTEM)照片, 间距为 $0.241 \mathrm{~nm} 、 0.264 \mathrm{~nm}$ 的清晰 晶格条纹应分别对应于 $\mathrm{Ag}_{3} \mathrm{PO}_{4}(211) 、(210)$ 晶面，相 应傅立叶变换(FFT)图(图 3(b)插图)显示的衍射斑点 为有序点阵结构, 表明 $\mathrm{Ag}_{3} \mathrm{PO}_{4}$ 纳米颗粒为结晶性 良好的单晶结构。

\section{3 不同磷酸银形貌形成机理分析}

反相微乳液法制备纳米材料, 其形貌结构与水 核直径大小密切相关, $W$ 值是决定水核大小的直接 因素; 此外, $W$ 值变化还会明显影响水核界面膜强 度，从而干扰水核相互接触时纳米粒子间的吸附、 堆砌以及聚集过程 ${ }^{[6,11]}$ 。基于上述理论, 本研究通 过改变 $W$ 值成功制备出形貌不同的系列体心立方 结构 $\mathrm{Ag}_{3} \mathrm{PO}_{4}$ 晶体材料, 其形成机理可以通过图 4 予 以解释: 当含有 $\mathrm{Ag}^{+}$与 $\mathrm{H}_{2} \mathrm{PO}_{4}^{-}$的微乳液滴相互接触 时, 油相融合后，水核中的 $\mathrm{Ag}^{+}$与 $\mathrm{H}_{2} \mathrm{PO}_{4}^{-}$发生化学 反应，生成 $\mathrm{Ag}_{3} \mathrm{PO}_{4}$ 晶核，晶核按照晶体各向异性生 长特性生成球形 $\mathrm{Ag}_{3} \mathrm{PO}_{4}$ 纳米晶粒子(图 3(b)); 当微 乳液体系中表面活性剂 SDS 数量不足使水核以球 形反胶团形态存在时，包裹着 $\mathrm{Ag}_{3} \mathrm{PO}_{4}$ 纳米晶粒子 的微乳液滴会进一步相互碰撞, 导致水核内纳米晶 粒子相互吸附、堆砌、聚集, 直至表面活性剂的数 量能够使水核以一定形态稳定存在，并使其对产物 形成“牢笼效应”阻止晶体进一步生长时，不同形貌 的 $\mathrm{Ag}_{3} \mathrm{PO}_{4}$ 纳米材料就得以形成(图 2(a d))。
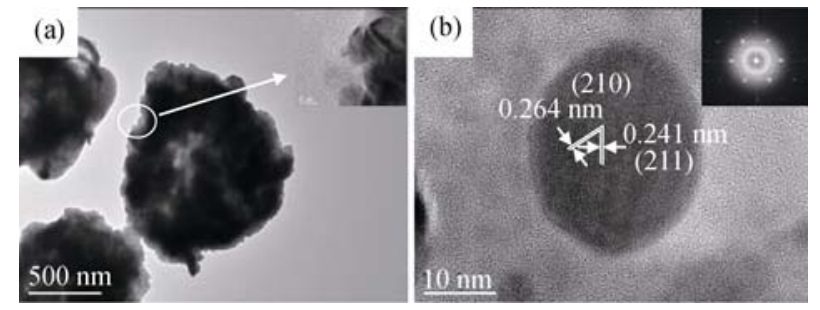

图 3 具有完整四面体形貌样品的 TEM 照片(a)与 $\mathrm{Ag}_{3} \mathrm{PO}_{4}$ 纳 米颗粒 HRTEM 照片(b)

Fig. 3 TEM images of the tetrahedral $\mathrm{Ag}_{3} \mathrm{PO}_{4}$ (a) and HRTEM image of $\mathrm{Ag}_{3} \mathrm{PO}_{4}$ nanoparticle (b) 


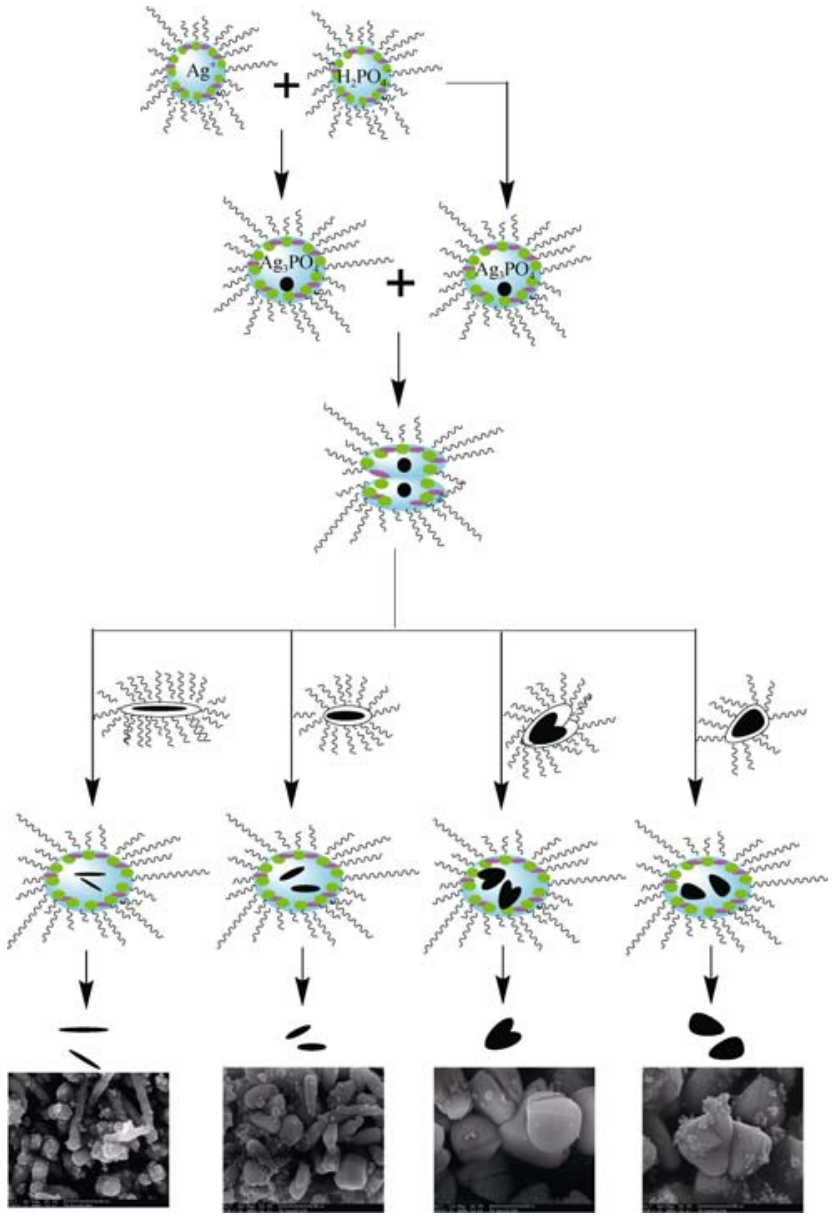

图 4 不同形貌 $\mathrm{Ag}_{3} \mathrm{PO}_{4}$ 产物形成机理示意图

Fig. 4 Size and morphology controlling mechanism of $\mathrm{Ag}_{3} \mathrm{PO}_{4}$ in microemulsions

onn : Surfactant molecule: O $n ?$ : Cosurfctant molecule

\subsection{UV-Vis-DRS 分析}

图 5(a)为不同形貌纳米 $\mathrm{Ag}_{3} \mathrm{PO}_{4}$ 材料紫外一可见 漫反射光谱(UV-Vis-DRS)图, 由图可知, 样品在可 见光区(400 750 nm)均有明显吸收, 说明实验产物

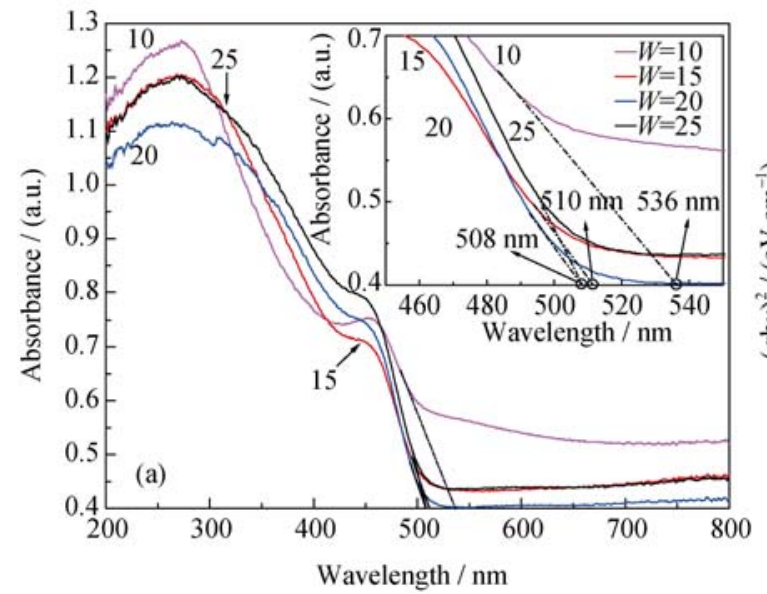

均有较好的可见光响应特性。棒状、纺锤状、类四 面体以及完整四面体形貌样品的吸收边分别位于 536、510、508 及 $508 \mathrm{~nm}$ (图 5(a)插图), 表明具有棒 状形貌的纳米 $\mathrm{Ag}_{3} \mathrm{PO}_{4}$ 可见光响应范围最大。

根据半导体带隙与带吸收边的关系, 通过 $(\alpha \mathrm{h} v)^{2}$ 对 $\mathrm{h} v$ 作图可估算出半导体带隙值 $\left(E_{\mathrm{g}}\right)$, 结果 如图 5(b)所示。 $W=10,15,20$ 及 25 制备的样品的带 隙值分别为 $2.31 、 2.43 、 2.44$ 与 $2.44 \mathrm{eV}$ 。样品微观 形貌变化对其带隙值的影响可通过材料量子限域效 应予以解释 ${ }^{[12-13]}$ 。当半导体材料尺寸减小时, 尤其 达到纳米量级时, 费米能级附近的电子能级由准连 续态向离散态转变，价带顶与导带底的位置均会发 生变化，从而影响材料带隙值。文献[14]中也发现了 相似的实验现象。

\section{5 光催化性能分析}

采用可见光催化降解次甲基蓝(MB)的浓度来评价 系列产物的光催化活性，结果如图 6(a)所示。本实验设 计的反相微乳体系制备的纳米 $\mathrm{Ag}_{3} \mathrm{PO}_{4}$ 对体积 $0.3 \mathrm{~L}$ 、 初始浓度 $10 \mathrm{mg} / \mathrm{L}$ 的 $\mathrm{MB}$ 均能够表现出高的光催化活 性, $40 \mathrm{~min}$ 内可催化降解 $90 \%$ 以上的 $\mathrm{MB}$ 。此外, 随着 纳米 $\mathrm{Ag}_{3} \mathrm{PO}_{4}$ 形貌由棒状向四面体状转变, 其光催化性 能逐渐增强。具有完整四面体形貌的 $\mathrm{Ag}_{3} \mathrm{PO}_{4}$ 表现出最 佳光催性能, $40 \mathrm{~min}$ 内 $\mathrm{MB}$ 的降解效率可达到 98\%(图 6(b))。Zheng 等 ${ }^{[15]}$ 认为, 具有四面体形貌 $\mathrm{Ag}_{3} \mathrm{PO}_{4}$ 的光 催化活性高与其能够裸露出更多的 $\{111\}$ 晶面有关，根 据 DFT 计算结果, $\mathrm{Ag}_{3} \mathrm{PO}_{4}\{111\}$ 晶面不仅表面能高 $(2.735 \mathrm{eV})$, 可以促进光催化反应在晶面上进行，而且 带隙值大 $(2.736 \mathrm{eV})$ ，也可以有效抑制光生电子与空穴 的复合，进而有效提高材料光催化性能。

为验证上述观点，本实验对比分析了纺锤状与 四面体状 $\mathrm{Ag}_{3} \mathrm{PO}_{4}$ 的 HRTEM 照片, 结果如图 7 所

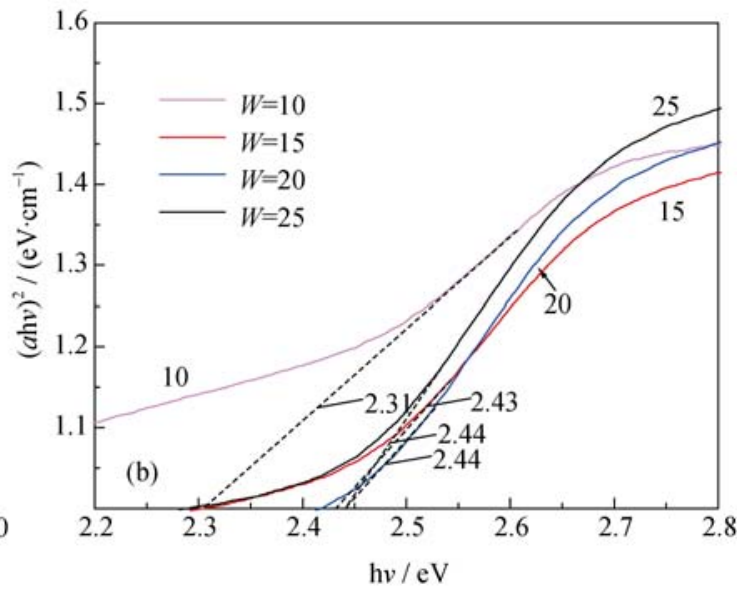

图 5 不同 $W$ (水与表面活性剂的比值)值下制备样品 UV-Vis 漫反射吸收谱(a)与 $(a \mathrm{~h} v)^{2}-\mathrm{h} v$ 图(b)

Fig. 5 UV-Vis diffuse reflectance spectra (a) and ( $a \mathrm{~h} v) v s \mathrm{~h} v$ plats (b) for different $W$ values samples 

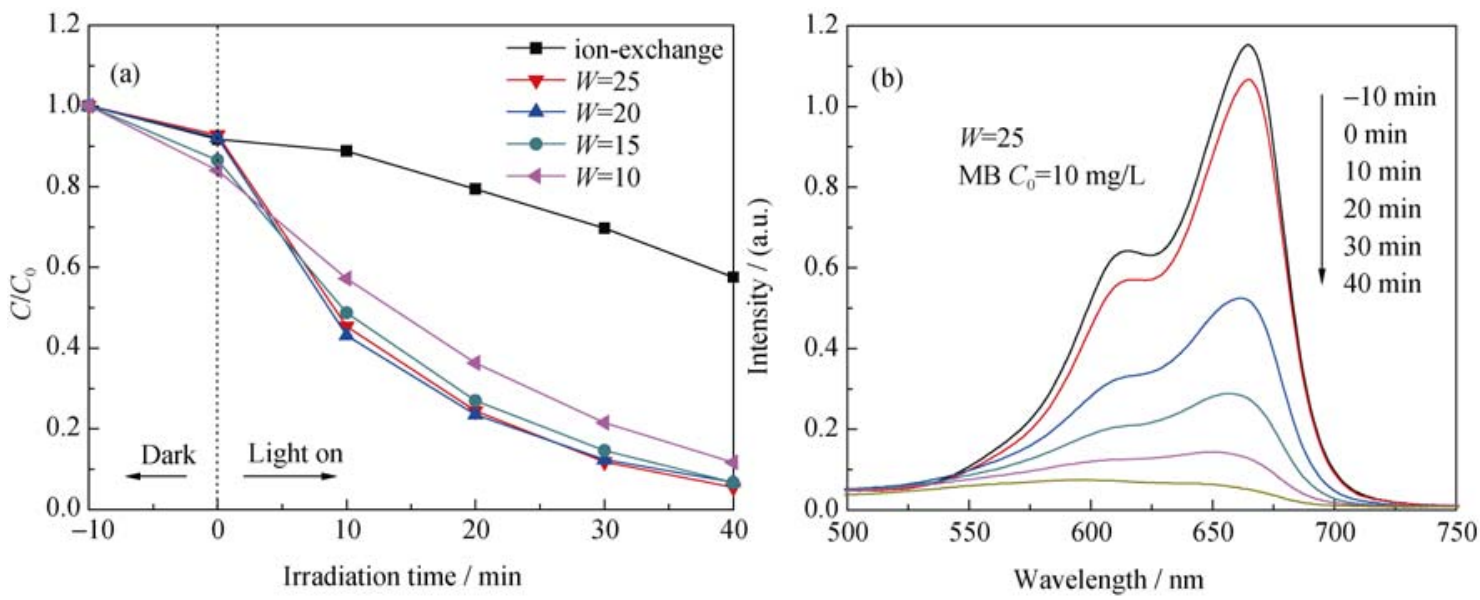

图 6 可见光照射下不同样品的光催化降解 $\mathrm{MB}$ 性能

Fig. 6 Photocatalytic degradation of MB over different samples prepared under visible-light irradiation with different $W$ values (a) and $W=25$ (b)
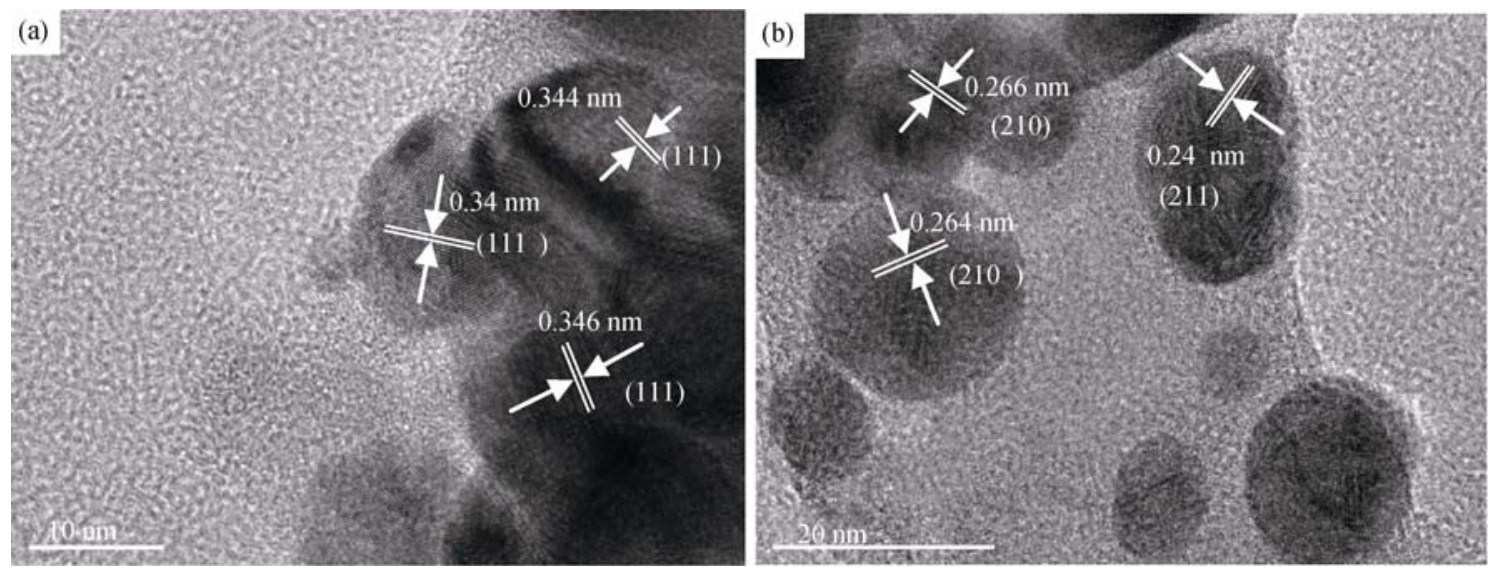

图 7 不同 $W$ (水与表面活性剂的比值)下制备 $\mathrm{Ag}_{3} \mathrm{PO}_{4}$ 的 HRTEM 照片

Fig. 7 HRTEM image of $\mathrm{Ag}_{3} \mathrm{PO}_{4}$ nanoparticle prepared with different $W$ values (a) $W=25$; (b) $W=15$

示。四面体状 $\mathrm{Ag}_{3} \mathrm{PO}_{4}$ 明显有较多的(111)晶面，而 在纺锤状样品中却很难发现(111)晶面。此外, 样品 比表面积测试结果表明, 四面体状样品比表面积 $\left(3.31 \mathrm{~m}^{2} / \mathrm{g}\right)$ 明显低于纺锤体样品 $\left(5.02 \mathrm{~m}^{2} / \mathrm{g}\right)$, 说明样 品比表面积变化并不是影响 $\mathrm{Ag}_{3} \mathrm{PO}_{4}$ 光催化性能的 关键性因素, 而样品能否裸露出更多的 $\{111\}$ 晶面 才是决定其光催化性能的主要因素。关于这一点在 Dong ${ }^{[3]}$ 与 $\mathrm{Hu}^{[16]}$ 等研究工作中也获得证实。

\section{3 结论}

以硝酸银、磷酸二氢钾为原料, 在 SDS/环己烷/ 异戊醇/水溶液的反相微乳液体系中制备了不同形貌 的纳米 $\mathrm{Ag}_{3} \mathrm{PO}_{4}$, 结论如下:

1) 实验制备的系列纳米 $\mathrm{Ag}_{3} \mathrm{PO}_{4}$ 均为结晶性能 良好的体心立方结构晶体材料;
2) 改变水与表面活性剂的比值 $(W)$ 可以调控制 备出棒状、纺锤状及类四面体、完整四面体状 $\mathrm{Ag}_{3} \mathrm{PO}_{4}$ 纳米材料。产物形貌不同主要由于 SDS 量 的变化改变了含有产物水核的直径与界面膜强度, 导致水核形状改变后影响产物形貌;

3) 实验制备的纳米 $\mathrm{Ag}_{3} \mathrm{PO}_{4}$ 均表现出好的可见 光响应特性及可见光催化降解 $\mathrm{MB}$ 性能, 其中, 具 有完整四面体形貌的 $\mathrm{Ag}_{3} \mathrm{PO}_{4}$ 光催化性能最佳，原 因与其能够裸露出更多的 $\{111\}$ 晶面有关。

\section{参考文献:}

[1] YI Z G, YE J H, NAOKI K, et al. An orthophosphate semiconductor with photooxidation properties under visible-light irradiation. Nat. Mater., 2010, 9(7): 559-564.

[2] BI Y P, OUYANG S X, UMEZAWA N, et al. Facet effect of single crystalline $\mathrm{Ag}_{3} \mathrm{PO}_{4}$ sub-microcrystals on photocatalytic properties. 
J. Am. Chem. Soc., 2011, 133(17): 6490-6492.

[3] DONG L H, WANG P, WANG S, et al. A simple way for $\mathrm{Ag}_{3} \mathrm{PO}_{4}$ tetrahedron and tetrapod microcrystals with high visible light-responsive activity. Mater. Lett., 2014, 134: 158-161.

[4] YANG Z M, TIAN Y, HUANG G F, et al. Novel 3D flower-like $\mathrm{Ag}_{3} \mathrm{PO}_{4}$ microspheres with highly enhanced visible light photocatalytic activity. Mater. Lett., 2014, 116: 209-211.

[5] WU A P, TIAN C G, CHANG W, et al. Morphology-controlled synthesis of $\mathrm{Ag}_{3} \mathrm{PO}_{4}$ nano/microcrystals and their antibacterial properties. Mater. Res. Bull. 2013, 48(9): 3043-3048.

[6] MALIK M A, WANI M Y, HASHIM M A. Microemulsion method: A novel route to synthesize organic and inorganic nanomaterials. Arab J. Chem., 2012, 5(4): 397-417.

[7] TENG HONG-HUI, XU SHU-KUN, WANG MENG, et al. Controllable synthesis of different dimensions nano- $\mathrm{ZnO}$ by microemulsion and photocatalytic activity. Journal of Inorganic Materials, 2010, 25(10): 1034-1040.

[8] DOMINGUEZ M S, MENDOZA G M, VARGAS M J R, et al. Synthesis of $\mathrm{Zn}$-doped $\mathrm{TiO}_{2}$ nanoparticles by the novel oil-in-water $(\mathrm{O} / \mathrm{W})$ microemulsion method and their use for the photocatalytic degradation of phenol. J. Environ. Chem. Eng., 2015, 3(4): 3037-3047.

[9] ABAZARI R, MAHJOUB A R, SAGHATFOROUSH L A, et al. Characterization and optical properties of spherical $\mathrm{WO}_{3}$ nanoparticles synthesized via the reverse microemulsion process and their photocatalytic behavior. Mater. Lett., 2014, 133: 208-211.

[10] DAS N, MAJUMDAR R, SEN A, et al. Nanosized bismuth ferrite powder prepared through sonochemical and microemulsion techniques. Mater. Lett., 2007, 61(10): 2100-2104.

[11] LANDFESTER K, MUSYANOVYCH A, MAILÄNDER A. From polymeric particles to multifunctional nanocapsules for biomedical applications using the minmulsion process. J. Polym. Sci. Pol. Chem., 2010, 48(3): 493-515.

[12] ZHANG Q, GONG W J, WANG J H, et al. Size-dependent magnetic, photoabsorbing, and photocatalytic properties of single-crystalline $\mathrm{Bi}_{2} \mathrm{Fe}_{4} \mathrm{O}_{9}$ semiconductor nanocrystals. J. Phys. Chem. C, 2011, 115(51): 25241-25246.

[13] NORRIS D J, BAWENDI M G. Measurement and assignment of the size-dependent optical spectrum in CdSe quantum dots. Phys. Rev. B, 1996, 53(24): 16338-16346.

[14] HE J, GUO R Q, FANG L, et al. Characterization and visible light photocatalytic mechanism of size-controlled $\mathrm{BiFeO}_{3}$ nanoparticles. Mater. Res. Bull., 2013, 48(9): 3017-3024.

[15] ZHENG B J, WANG X, LIU C, et al. High-efficiently visible light-responsive photocatalysts: $\mathrm{Ag}_{3} \mathrm{PO}_{4}$ tetrahedral microcrystals with exposed $\{111\}$ facets of high surface energy. J. Mater. Chem. A, 2013, 1(40): 12635-12640.

[16] HU H Y, JIAO Z B, YU H C, et al. Facile synthesis of tetrahedral $\mathrm{Ag}_{3} \mathrm{PO}_{4}$ submicro-crystals with enhanced photocatalytic properties. J. Mater. Chem. A, 2013, 1(7): 2387-2390. 